\title{
Exercício físico e o processo saúde-doença no envelhecimento
}

\author{
Physicd exerciseandhelth-dseprocessineddely
}

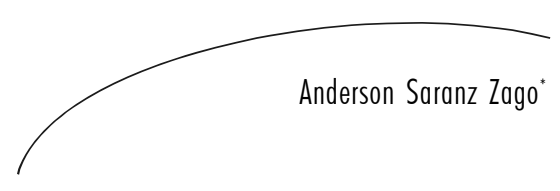

\section{Resumo}

Juntamente com o crescente número de idosos na população, tem-se observado significativo aumento na incidência de várias doenças, como hipertensão arterial, osteoporose e diabetes, que comprometem a qualidade de vida e autonomia dos idosos. Em contrapartida, as mudanças no estilo de vida, incluindo especialmente alimentação saudável e exercício físico, assumem papel de destaque na relação saúde-doença, pois diversos estudos apontam para os benefícios dessas mudanças na diminuição da incidência e prevenção de várias doenças. No entanto, mesmo com o conhecimento dos benefícios que a prática regular de exercícios físicos gera para a saúde e com diversas campanhas de incentivo à prática regular de exercícios físicos, os níveis de sedentarismo ainda permanecem elevados na população brasileira. Desta forma, incentivar campanhas que priorizam as mudanças no estilo de vida são atitudes acertadas para gerar melhor qualidade de vida à população e sobretudo para o fortalecimento da relação saúde-doença, especialmente por intermédio do exercício físico.

\section{Abstract}

Along with the growing number os elderly in the population, there is a significant increase in the incidence of several diseases, such as hypertension, osteoporosis and diabetes, which affect the quality of life and independence of the elderly. However, lifestyle changes, including healthy food and physical exercise, may be considered an important factor for the relationship between health and disease. Many studies have shown that lifestyle changes may decrease the incidence of many diseases; however the benefits of the physical exercises seem to be often ignored. Although many advertising campaigns show the benefit of physical exercise for a healthy lifestyle, the proportion of sedentary individuals is still high. Thus, changes in lifestyle and physical exercise should be encouraged for the elderly population, in order to reduce the incidence of disease and improving the relationship between health and disease and, as a consequence, the quality of life in this population.

\footnotetext{
Universidade de São Paulo. Escola de Educação Física e Esportes de Ribeirão Preto. Ribeirão Preto, SP, Brasil

Correspondência / Correspondence

Anderson Saranz Zago

Universidade de São Paulo. Escola de Educação Física e Esportes de Ribeirão Preto.

Av Bandeirantes, $\mathrm{n}^{\circ} 3900$ - Monte Alegre

14040-900 - Ribeirão Preto, SP, Brasil

E-mail: aszago@usp.br
}

Palavras-chave: Atividade Motora. Envelhecimento. Qualidade de Vida. Doença. Saúde do Idoso. Exercício Físico.

Key words: Motor Activity. Aging. Quality of Life. Disease. Health of the Elderly. Physical Exercise. 
INTRODUÇÃO

A busca por medidas objetivando uma melhor qualidade de vida, especialmente na população idosa, tem sido o foco de muitas pesquisas. É consenso na literatura que há uma relação muito forte entre envelhecimento, estilo de vida e saúde, e que a prática regular de exercícios físicos assume papel de destaque nesta relação. Desta forma, este texto tem como finalidade discutir tais relações, caracterizando o exercício físico como um dos principais fatores na relação saúde-doença e na melhoria da qualidade de vida, especialmente entre as pessoas acima de 60 anos de idade.

\section{Exercício físico e o processo saúde-doença no envelhecimento}

Tem-se observado, atualmente, uma alteração drástica na pirâmide etária tanto da população mundial quanto da brasileira. Dados do IBGE ${ }^{1}$ mostraram que a população idosa em 1991 representava 7,3\% da população total do Brasil. No ano de 2000, esse número passou para $8,6 \%$ e a expectativa para 2020 seria de $13 \%$ de idosos.

Por definição, envelhecimento é a somatória de todos os processos (alterações e adaptações) que ocorrem com o passar dos anos no organismo humano. ${ }^{2}$ Esta é somente uma das várias definições existentes hoje na literatura que fala a respeito do envelhecimento e da pessoa idosa. Envelhecer é um processo natural que ocorre desde o nascimento até a morte, e que é caracterizado por uma série de alterações inerentes ao processo natural de envelhecimento, principalmente a partir da sexta década de vida.
Dentre essas alterações, pode-se citar como exemplo a diminuição da capacidade funcional devido à diminuição de alguns componentes de capacidade física como força muscular, resistência aeróbia e coordenação, diminuição da acuidade visual e aumento da incidência de doenças, principalmente as cardiovasculares. ${ }^{3-5}$

Com os avanços tecnológicos, especialmente na área médica, esperar-se-ia um aumento significativo na qualidade de vida dos idosos, mas pelo contrário, essa enorme alteração no quadro demográfico está sendo acompanhada por um aumento significativo da incidência de determinadas doenças, que além de diminuir a expectativa de vida do idoso, gera uma condição de dependência e baixa autoestima. ${ }^{5,6}$ Veras $^{7}$ apontou em seu estudo que há uma relação de proporcionalidade entre aumento da idade e aumento da prevalência de doenças crônicas, evidenciando que na população, a cada três indivíduos, um é portador de doença crônica e, entre os idosos, oito em cada dez possuem pelo menos uma doença crônica.

Além de comprometer a pessoa idosa propriamente dita, esses problemas relacionados à saúde acabam gerando várias consequências para todos os setores, como aumento nos gastos com os sistemas de saúde pública. Silvestre et al., ${ }^{8}$ ao dividirem a população em três grupos distintos ( 0 a 14 anos, 15 a 59 anos e 60 anos ou mais), analisaram variáveis como índice de mortalidade, custo e internações em hospitais, e concluíram que a população de idosos estaria gerando um gasto significativamente maior para os sistemas de saúde, quando comparada aos outros dois grupos (tabela 1). 
Tabela 1 - Coeficiente de hospitalização, custo hospitalar e média de permanência em hospitais da população brasileira. Ribeirão Preto, SP, 2009.

\begin{tabular}{|c|c|c|c|}
\hline & 0 a 14 anos & 15 a 59 anos & 60 anos ou mais \\
\hline \multicolumn{4}{|l|}{ Coeficiente de Hospitalizações } \\
\hline \multicolumn{4}{|l|}{ Número de hospitalizações por 1000} \\
\hline habitantes & 46 & 79 & 165 \\
\hline \multicolumn{4}{|l|}{ Índice de custo Hospitalar } \\
\hline \multicolumn{4}{|l|}{ Custo de hospitalização por habitante / ano } \\
\hline & $\mathrm{R} \$ 10,93$ & $\mathrm{R} \$ 18,48$ & $\mathrm{R} \$ 55,25$ \\
\hline Média de Permanência & & & \\
\hline $\begin{array}{l}\text { Número médio de dias hospitalizado / } \\
\text { habitante }\end{array}$ & 5,1 & 5,1 & 6,8 \\
\hline
\end{tabular}

Adaptado de Silvestre et al., (1999). ${ }^{8}$

De acordo com a tabela 1, o idoso, além de ter um alto índice de mortalidade com relação às outras faixas etárias, apresenta maior gasto diário no hospital e maior número de dias internado. $\mathrm{Na}$ realidade, esses dados são somente um exemplo da seriedade em que a questão do envelhecimento deve ser encarada nos dias atuais.

A Lei ${ }^{\circ} 8842 / 94^{9}$ e o Decreto ${ }^{\circ} 1948 / 96,{ }^{10}$ da Política Nacional do Idoso asseguram o direito à pessoa idosa e recomendam a criação de condições para a promoção de autonomia, integração e participação efetiva na sociedade, reafirmando o direito à saúde nos diversos níveis de atendimento. Desta forma, os profissionais da área de saúde têm o dever ético, moral e cívico de adotar atitudes que privilegiem o idoso, não no sentido direto de diminuição de gastos com saúde, mas principalmente focando na saúde do idoso, garantindo ou dando condições de autonomia e melhor qualidade de vida. Dentre as diversas formas para se atingir este fim, o exercício físico tem-se mostrado extremamente eficaz para a relação saúde-doença, sobretudo quando se leva em consideração o conceito de saúde como um bem-estar físico, social e mental.

Um dos principais problemas de saúde que também é apontado como um dos principais fatores de mortalidade para a população idosa são as doenças cardiovasculares. ${ }^{11,12}$ Bolkovoy \& Blair ${ }^{11}$ mostraram que a mudança no estilo de vida representaria $54 \%$ de diminuição da taxa de mortalidade por doenças coronarianas, enquanto que a intervenção médica seria responsável por $39,5 \%$ da taxa de declínio e outras causas, por apenas $6,5 \%$. $\mathrm{Na}$ realidade, o que esse estudo sugere é que a adoção de um estilo de vida adequado, incluindo alimentação balanceada e prática regular de exercícios físicos, pode contribuir para diminuir a incidência de várias doenças, e principalmente elevar a qualidade de vida da população idosa.

Na revisão bibliográfica realizada por Gobbi, Villar e Zago ${ }^{13}$ qualquer indivíduo que adotar um estilo de vida ativo, abolindo definitivamente o sedentarismo, terá uma diminuição de $40 \%$ no risco de morte por doenças cardiovasculares. Infelizmente, no Brasil há uma estimativa de que 60 a $70 \%$ da população não participam de programas regulares de exercício físico ${ }^{1,14,15,16} \mathrm{e}$, de acordo com Spirduso, ${ }^{2} 50 \%$ do declínio biológico são provocados pela atrofia por desuso resultante da falta de exercício físico. Esses dados justificam a alta incidência de doenças na população idosa brasileira.

Assim, manter-se fisicamente ativo é uma atitude que irá beneficiar a relação saúde-doença 
e provavelmente alteraria muitos dos dados epidemiológicos atuais.

A ideia de que o exercício físico possui relação direta na temática saúde-doença parece já estar concretizada. Resta saber o que realmente o exercício físico promove no organismo humano para que ocorra uma diminuição da incidência de doença e, consequentemente, aumento nos níveis da saúde, especialmente da população idosa, promovendo assim a conscientização da população da real importância do exercício físico regular no âmbito da saúde.

Dependendo do tipo e forma de exercício a ser realizado e do problema que se pretende combater, os efeitos benéficos desta prática regular serão diretos ou indiretos para a qualidade de vida dessas pessoas. ${ }^{17} \mathrm{Um}$ bom exemplo seria o mal de Parkinson e Alzheimer, em que o exercício físico teria efeito indireto, pois traria uma grande contribuição para minimizar os efeitos e sintomas dessas doenças, e não na doença propriamente dita. ${ }^{18}$ Por outro lado, o exercício físico possui efeito direto em várias outras doenças e pode ser considerado como um preditor de saúde, como é o caso das doenças cardiovasculares.

Como dito anteriormente, as doenças cardiovasculares são consideradas a principal causa de morte, especialmente na população idosa. ${ }^{12,17}$ Os principais fatores de risco para as doenças cardiovasculares, de acordo com Liberman ${ }^{19} \mathrm{e}$ Pereira et al. ${ }^{20}$ são o sedentarismo, hipertensão arterial e níveis elevados de LDL-colesterol. Se for utilizado como exemplo apenas a hipertensão arterial, existe uma alta incidência desta patologia em indivíduos entre 65 a 74 anos $(60,9 \%$ em homens e $74 \%$ nas mulheres), justificando assim a alta mortalidade por essa doença. ${ }^{19}$

A hipertensão arterial é uma doença crônica degenerativa de origem multifatorial, ou seja, diversos fatores podem estar envolvidos em sua etiologia como, por exemplo, fatores genéticos, ambientais, estresse, dentre outros. Em termos fisiológicos, quando a hipertensão arterial é diagnosticada no indivíduo, podem-se observar várias alterações orgânicas que são responsáveis pelo aumento da pressão arterial. Essas alterações são: o aumento do débito cardíaco; aumento da atividade nervosa simpática; aumento da resistência periférica; aumento do volume sanguíneo, devido a uma maior retenção líquida; aumento da ativação do sistema reninaangiotensina, responsável pela vasoconstrição; diminuição da produção e liberação de óxido nítrico, um potente vasodilatador; diminuição da expressão de determinados genes responsáveis pela produção de proteínas que atuam no controle cardiovascular; dentre outros. ${ }^{12,21,22}$

$\mathrm{Na}$ realidade, o importante é que se tenha o conhecimento de que o exercício físico pode atuar em cada um desses fatores, contribuindo para a diminuição dos valores de pressão arterial. Como exemplo, pode-se citar o aumento da liberação de óxido nítrico com o exercício. Durante a realização de qualquer exercício, percebe-se que há aumento da frequência cardíaca e do fluxo sanguíneo, que culmina num aumento do shear stress, que é a força em que o sangue "raspa" na parede arterial. Essa força é captada por mecanoreceptores que desencadeiam uma série de reações químicas, dentre elas a ativação de uma enzima responsável pela produção de óxido nítrico. Por ser um potente vasodilatador, o óxido nítrico aumenta o calibre do vaso sanguíneo, diminuindo assim a resistência periférica. ${ }^{23,24}$ Este simples mecanismo é capaz de diminuir os valores de pressão arterial e, consequentemente, a incidência de hipertensão arterial.

Este exemplo deixa claro que o exercício físico presta enorme contribuição para favorecer a saúde da população idosa, com a diminuição ou minimização da doença ou dos efeitos que ela causa. $\mathrm{Na}$ realidade, existe uma série de estudos que ressaltam os benefícios do exercício físico para com a saúde, mostrando melhoras nos quadros de hipertensão arterial, diabetes, osteoporose e significativo aumento na qualidade de vida dessas pessoas. ${ }^{12,25-27}$

Desta forma, o exercício físico pode e deve ser encarado como uma das principais ferramentas do processo saúde doença, pois a contribuição que ele traz beneficia tanto o 
indivíduo idoso, principal alvo para esta relação, quanto o poder público com os gastos no sistema público de saúde.

Apesar de todas essas evidências, os níveis de sedentarismo ainda permanecem extremamente elevados. Estudos apontam que aproximadamente $60 \%$ da população brasileira são considerados sedentários ${ }^{13,16}$ e a relação entre idosos, altos índices de doenças e sedentarismo pode estar contribuindo para a diminuição da qualidade de vida das pessoas idosas.

Diversas campanhas são promovidas no intuito de combater o sedentarismo, gerando benefícios para a saúde, qualidade de vida e também benefícios econômicos. No cenário da atividade física, uma das maiores campanhas nacionais de incentivo à prática regular de atividade física é o AGITA São Paulo (Campinas, Santos etc.). Nesta campanha, ou neste programa, há um incentivo muito forte para a melhoria da qualidade de vida e autonomia, através da prática regular de exercícios físicos com a proposta de acúmulo de 30 minutos diários de qualquer atividade física (esportiva, lazer, doméstica etc.).

Outra campanha que merece destaque é a "Entre para o time onde a atividade física e o meio ambiente jogam juntos", promovida pelo Ministério da Saúde. Esta campanha, lançada no dia 4 de abril de 2008, em comemoração ao Dia Mundial da Saúde (7 de abril), tem como foco principal a preservação do meio ambiente, a prática regular de atividades físicas, o planejamento e acesso a locais públicos como praças, hortos florestais etc. e a promoção de saúde. O Programa Saúde da Família (PSF), criado pelo Ministério da Saúde em 1994, também merece destaque, por envolver uma equipe multidisciplinar para os cuidados com a saúde da população, incluindo aí o profissional de educação física e, consequentemente, o exercício físico.
Na realidade, essas campanhas são um reflexo tanto da Política Nacional do Idoso, da Política Nacional de Saúde da Pessoa Idosa, quanto do Estatuto do Idoso, que são dispositivos legais que norteiam as ações sociais e de saúde, garantindo os direitos da pessoa idosa a um envelhecimento ativo e com manutenção da autonomia. Tais ações envolvem a diminuição de doenças, aumentando a capacidade funcional e mental e o engajamento social.

Esta descrição está de acordo com o Estatuto do Idoso, que no artigo 18, capítulo IV, relata que as instituições de saúde devem obedecer aos critérios mínimos para o atendimento do idoso em termos físicos, sociais e mentais.

\section{CONSIDERAÇÕES FINAIS}

Todas as alterações que objetivamos com relação aos dados epidemiológicos e que fortalecem a relação saúde-doença, na realidade dependem de nós mesmos. À desinformação, ao preconceito e ao desrespeito aos idosos somam-se a precariedade de investimentos públicos para atendimento às necessidades específicas desta população, e mesmo de recursos humanos, tanto em qualidade quanto em quantidade. ${ }^{7}$

As leis existem e seus conteúdos são bastante abrangentes, englobando todo o universo do envelhecimento. Basta cumprir e exigir que os órgãos administrativos cumpram as leis, exigindo locais adequados, equipamentos e sobretudo pessoal capacitado, para que o idoso tenha uma vida digna e com qualidade de vida.

$\mathrm{O}$ incentivo a campanhas que visem principalmente à conscientização da população para a adoção a estilos de vida mais saudáveis também é atitude que somente trará benefícios tanto para a qualidade de vida quanto para a autonomia da pessoa idosa, fortalecendo a relação saúde-doença por intermédio do exercício físico. 


\section{REFERÊNCIAS}

1. IBGE. Pesquisa sobre o padrão de vida brasileiro. Rio de Janeiro: IBGE; 2004. Disponível em: URL: www.ibge.gov.br/ imprensa/ notícias/ppv11.html

2. Spirduso WW. Physical dimensions of aging. Champaign: Human Kinetics. 1995. 432 p.

3. American College of Sports Medicine. Atividade física, aptidão física e hipertensão arterial. Revista Brasileira de Medicina do Esporte 1998 set./out; 4(5): 160-7.

4. Brum PC, et al. Adaptações agudas e crônicas do exercício físico no sistema cardiovascular. Revista Paulista de Educação Física 2004 ago; 18(n. especial): 21-31.

5. Carvalho JAM, Garcia RA. O envelhecimento da população brasileira: um enfoque demográfico. Caderno de Saúde Pública 2003 mai./jun; 19(3): 725-33.

6. Chaimowicz F. A saúde dos idosos brasileiros às vésperas do século XXI: problemas, projeções e alternativas. Rev Saúde Pública 1997 abr; 31(2): 184-200.

7. Veras R. Envelhecimento populacional contemporâneo: demandas, desafios e inovações. Rev Saúde Pública. 2009; 43(3): 548-54.

8. Silvestre JA. Área técnica da saúde do idoso. Ministério da Saúde. Brasília 1999.

9. Brasil. Lei n ${ }^{\circ} 8842$ de 4 de Janeiro de 1994. Dispõe sobre a Política Nacional do Idoso, cria o Conselho Nacional do Idoso e dá outras providências. Pub DOU, [1994 jan 05].

10. Brasil. Decreto ${ }^{\circ} 1948$ de 3 de Julho de 1996. Regulamenta a Lei ${ }^{\circ} 8842$, de 4 de Janeiro de 1994, que dispõe sobre a Política Nacional do Idoso, e dá outras providências. Pub DOU, [1996 jul 04].

11. Bolkovoy JL, Blair SN. Aging and exercise: a health perspective. J Aging Phys Act 1994; 2(3): 243-60.

12. Pescatello LS, et al. American College of Sports Medicine position stand: exercise and hypertension. Med Sci Sports Exerc 2004; 36(3): 533-53.

13. Gobbi S, Villar R, Zago AS. Bases teóricopráticas do condicionamento físico. Rio de Janeiro: Guanabara Koogan. 2005. Educação Física no Ensino Superior. 284 p.
14. Marques AT e Gaya A. Atividade física, aptidão física e educação para a saúde: estudos na área pedagógica em Portugal e no Brasil. Revista Paulista de Educação Física 1999 jan./jun; 13(1): 83-102.

15. Trombetta IC, et al. Sedentarismo e risco cardiovascular. Hipertensão 2006; 9(2): 65-71.

16. Marin MJS, et al. A atenção à saúde do idoso: ações e perspectivas dos profissionais. Revista Brasileira de Geriatria e Gerontologia 2008; 11(2): 245-58.

17. Oliveira GM, Klein CH, Souza e Silva NA. Mortality from cardiovascular diseases in three Brazilian states from 1980 through 2002. Rev Panam Salud Publica 2006 feb; 19(2): 85-93.

18. Tanaka $\mathrm{K}$, et al. Benefits of physical exercise on executive functions in older people with Parkinson's disease. Brain Cogn 2009 mar; 69(2): 435-41.

19. Liberman A. Aspectos epidemiológicos e o impacto clínico da hipertensão no indivíduo idoso. Revista Brasileira de Hipertensão 2007 jan./mar; 14(1): 17-20.

20. Pereira RBR, et al. O Impacto dos fatores de risco cardiovascular sobre a função e a estrutura vascular e cardíaca. Hipertensão 2006; 9(1): 21-6.

21. Ortega KC, Mion Júnior D. Hipertensão arterial refratária. Hipertensão 2007; 10: 46-50.

22. Araújo CSC, Silva YL, Barreto-Filho JAS. Hipertensão arterial resistente. Hipertensão 2006; 9(1): 15-20.

23. Schiffrin EL. Vascular endothelin in hypertension. Vascul Pharmacol 2005 jun; 43(1): 19-29.

24. Zago AS, Zanesco A. Nitric oxide, cardiovascular disease and physical exercise. Arq Bras Cardiol 2006 dec; 87(6): 264-70.

25. Forjaz CLM, et al. Exercício físico e hipertensão arterial: riscos e benefícios. Hipertensão 2006; 9(3): 104-12.

26. Higashi Y, Yoshizumi M. Exercise and endothelial function: role of endotheliumderived nitric oxide and oxidative stress in healthy subjects and hypertensive patients. Pharmacol Ther 2004 apr; 102(1): 87-96.

27. Matsudo MM, Matsudo VKR, Barros Neto TL. Impacto do envelhecimento nas variáveis antropométricas, neuromotoras e metabólicas da aptidão física. Revista Brasileira de Ciências e Movimento 2000; 8(4): 21-32. 